\title{
LOS CICLOS EN EL DESARROLLO SOCIOCULTURAL EN MESOAMÉRICA: ESTUDIOS DE CASO EN LAS TIERRAS BAJAS MAYAS ${ }^{1}$
}

\author{
Stanislaw Iwaniszewski
}

Museo Arqueológico Estatal, Varsovia, Polonia

\begin{abstract}
ABstrucr: The author applies the concepts of dissipative structures and punctuated equilibrium to the study of four Maya sites (Uaxactun, Altar de Sacrificios, Seibal, and Piedras Negras). The stability of a sociocultural system is measured against rhythms of innovation occurrences. At each of the sites long periods with very few innovations are observed which attest for system's major stability and short periods with numerous innovations wich denote its major instability. Each site displays its own cycle of stability/instability periods.
\end{abstract}

Resumen: El autor aplica los conceptos de estructuras disipativas y del equilibrio punteado al estudio de cuatro sitios mayas (Uaxactún, Altar de Sacrificios, Seibal y Piedras Negras). La estabilidad del sistema sociocultural se mide por el ritmo de aparición de las innovaciones. En cada sitio se observan periodos largos con pocas innovaciones, que sugieren la mayor estabilidad del sistema local y periodos cortos con numerosas innovaciones que denotan su menor estabilidad. Cada uno de los sitios muestra su ciclo de los periodos de estabilidad/inestabilidad.

\section{Introducción}

La aplicación del concepto de estructuras disipativas a la investigación arqueológica puede contribuir a nuestro conocimiento del porqué de los cambios culturales. El fenómeno de la autoorganización del sistema sociocultural que ocurre cuando éste se encuentra lejos del equilibrio, y la hipótesis del equilibrio punteado, según la cual los largos periodos de stasis (saturación) son separados por los cortos, durante los cuales ocurren numerosas innovaciones, hacen suponer que el ritmo de aparición de innovaciones puede ser un criterio para evaluar su estabilidad en general. Es decir, deberían suceder más innovaciones en condiciones de desequilibrio del sistema sociocultural que durante los periodos en que éste se halla en un estado uniforme, estable. Puede sugerirse que la estabilidad del sistema sociocultural puede medirse por el ritmo de sus innovaciones.

Utilizando este enfoque, quisiera presentar los cambios en la evolución sociocultural de cuatro sitios mayas. Sin embargo, quisiera senalar que los procesos del cambio, aunque semejantes desde una perspectiva global, fueron diferentes en cada uno de estos sitios.

\footnotetext{
${ }^{1}$ Este trabajo fue presentado como ponencia en el Congreso Internacional de Antropología e Historia, celebrado en Veracruz, Veracruz, del 8 al 12 de septiembre de 1992.
} 


\section{Innovación y estabilidad cultural}

La innovación puede definirse como la formación de un rasgo, elemento, componente o estructura nueva. Aunque las innovaciones ocurren en cada sociedad humana, el ritmo de sus apariciones y la razón difieren. Lenski y Lenski (1974: 62-66) enumeran muchas causas de estas variaciones, lo que puede resumirse como: la capacidad del procesamiento de la información, las influencias externas y la actitud social hacia las innovaciones. No obstante, son éstas las causas específicas, originadas, en última instancia, por la falta de estabilidad del sistema.

Ya que los fenómenos de difusión de nuevas ideas o de innovaciones tecnológicas tienen efectos acumulativos (e.g. Lenski y Lenski, 1974: 67-68; Starr y Rudman, 1973), se grafican las curvas del crecimiento acumulativo de innovaciones para presentar su difusión (Lenski y Lenski, 1974: 65; Ammerman y Cavalli-Sforza, 1973; Cavalli-Sforza y Feldman, 1981: 29-30). En este ensayo utilizaré también este método porque lo que aquí es relevante es la interpretación de la forma de la curva.

Siguiendo a Price (1978) y a Crane (1972: 12 16, fig. 1), se puede dividir una curva logística en cuatro partes: el arranque del crecimiento, el crecimiento acelerado (exponencial), el crecimiento lento y el estancamiento (o la saturación). Por otra parte, se puede presentar la escala vertical (eje Y) en forma logarítmica, dejando la escala horizontal (eje X) en forma aritmética (e.g. Hamblin et al., 1973) y entonces la fase del crecimiento exponencial (acelerado) se grafica como una línea recta inclinada, mientras que la fase del crecimiento lento o saturado, en una línea poco inclinada. Las inclinaciones de ambas líneas se describen matemáticamente por ecuaciones correspondientes. En este trabajo lo que me interesa es determinar las cuatro fases mencionadas y estudiar las ubicaciones de pun-

Estudios de Cultura Maya. Vol. XXI, 2000

Instituto de Investigaciones Filológicas/

Centro de Estudios Mayas, UNAM

ISSN: 0185-2574 tos de inflexión, y por eso presento la escala vertical (eje Y) en forma aritmética. Obviamente, en vez de gráficas se pueden ofrecer series de ecuaciones diferenciales. En este trabajo adjunto solamente las series de razones de cambio (rates of change) en los cuadros que resumen los datos sobre innovaciones y por lo tanto no presento gráficas.

Tomando en cuenta la hipótesis de que suceden más innovaciones cuando el sistema sociocultural se encuentra en un estado relativamente menos estable, puede sugerirse que la parte de la curva logística que equivale a la etapa de saturación se refiere al estado más estable de dicho sistema. Entonces, la parte del arranque del crecimiento que se aproxima a la forma exponencial describe el estado de disipación y el punto de inflexión entre la parte que corresponde a la saturación y este arranque puede aproximarse al punto de bifurcación (véase en Luenberger, 1979: 320-321). De este modo, la curva que describe el crecimiento acumulativo de innovaciones (nuevos elementos) por fases temporales se refiere, de manera aproximada, a la trayectoria trazada por distintos estados del sistema en cuestión.

Para fines de este trabajo basta comparar las dos fases consecutivas referentes a la misma categoría de innovaciones para ver si la fase posterior sigue la misma tendencia que en la anterior o si hay un cambio. Estos cambios se definen por la presencia de puntos de inflexión en las curvas. Si la misma tendencia sigue por lo menos por el lapso de dos fases consecutivas, ambas se encuentran del mismo lado del punto de inflexión. Cuando las tendencias cambian, pasan de un lado del punto de inflexión al otro. La fase del arranque del crecimiento y del crecimiento acelerado se encuentran a un lado del punto de inflexión, mientras que las fases del crecimiento lento y del estancamiento están en el otro. 
Ahora bien, se elige un número de diferentes variables que se refieren a los distintos subsistemas culturales y se calculan las innovaciones por fases culturales definidas arqueológicamente. Las curvas acumulativas de las innovaciones mostrarán una serie de formas sigmoides exponiendo cierto número de puntos de inflexión. Para determinar en cuál fase hubo más cambios de tendencias, simplemente he dividido cada punto de inflexión entre dos (ya que cada punto de transición se refiere a dos fases) y el resultado lo adscribo a cada dos fases. Ya que cada fase colinda con otras dos (salvo la primera fase y la última), el valor final de los cambios se describe sumando las cantidades de puntos de inflexión con respecto a la fase anterior y posterior. Como resultado de esto, cada fase se caracteriza por un número que describe las tendencias en los cambios de puntos de inflexión. Para obtener las razones de cambios basta dividir esta cantidad entre el periodo que dura la fase. Las fases con mayor número de razones de cambios se pueden considerar como las que tienen menor estabilidad en comparación con las fases vecinas. De esta manera, grosso modo defino las fases de la trayectoria que corresponden a los periodos de mayor o menor estabilidad.

Otro problema importante relacionado con las innovaciones es el llamado efecto multiplicador (Lenski y Lenski, 1974: 67, y en especial Renfrew, 1984b: 269-276, 1984a; Renfrew y Cooke, 1984), según el cual una innovación con frecuencia origina otras en cadena y hace que las innovaciones en un subsistema actúen en favor de otras innovaciones en otros subsistemas. Renfrew (1984b: 276), incluso, observa que un solo factor no puede producir cambios en la estructura de la cultura; se necesitan aquí las innovaciones que producen cambios en, por lo menos, dos diferentes subsistemas. Se puede notar la semejanza entre el fenómeno del efecto multiplicador y la hipótesis del equilibrio punteado, aunque son explicados de diferente manera.

Las variables escogidas describen, de algún modo, las trayectorias de más de un subsistema, pero pueden estudiarse las variables que pertenecen a diferentes niveles de complejidad dentro del mismo subsistema y entonces hay que juzgar su representatividad. Naturalmente hay muchos más problemas relacionados con el estudio de innovaciones no enumerados aquí por razones de espacio. Disponiendo de un número de datos que describen la trayectoria de un subsistema, prefiero hablar solamente en términos de mayor o menor estabilidad; los casos concretos deberían mostrar las limitaciones y ventajas de este enfoque.

\section{Cerámica}

Tomando en cuenta la aparición de las innovaciones relacionadas con la cerámica se nota que existen los periodos cuando se observa un crecimiento de innovaciones lento $\mathrm{y}$, asimismo, hay periodos en los que éste se vuelve acelerado. Las trayectorias referentes al crecimiento acumulativo de innovaciones muestran dos o tres ciclos que se repiten en cada uno de los sitios investigados.

En Uaxactún se pueden separar las etapas siguientes (véase el cuadro 1): el crecimiento de innovaciones en Mamón está seguido por una tendencia al estancamiento en Chicanel. Una tendencia de arranque del crecimiento se observa en Tzakol 1, lo que se transforma en la fase de crecimiento acelerado en Tzakol 2 y termina con una etapa nueva de la saturación en Tzakol 3. En Tepeu 1 se observa un nuevo arranque del crecimiento seguido por las fases de crecimiento acelerado, sobre todo en Tepeu 3.

Analizando este fenómeno desde cerca (compárense los cuadros 5 a y 5b) se observan las si- 


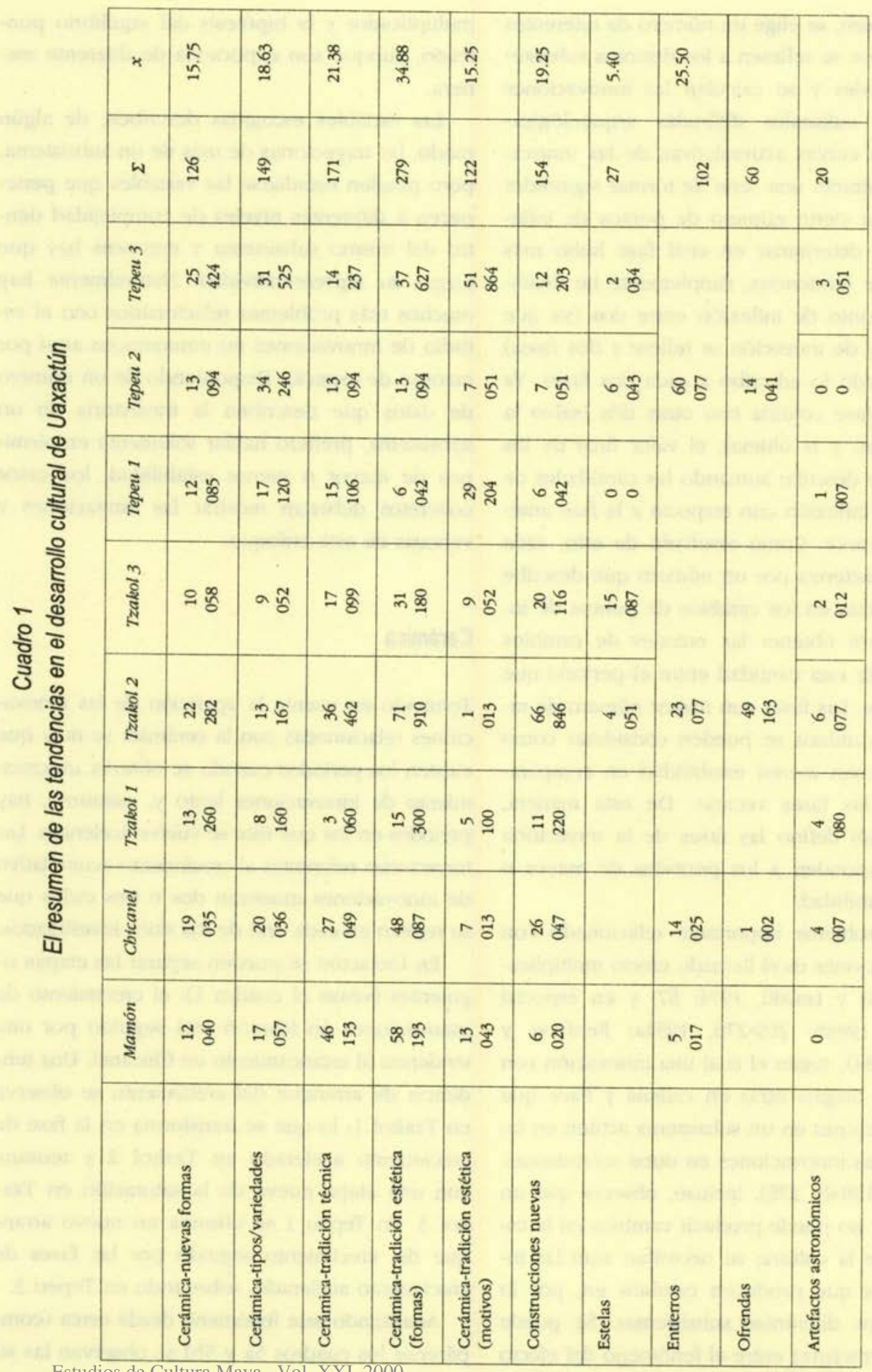

Estudios de Cultura Maya. Vol. XXI, 2000

Instituto de Investigaciones Filológicas/

Centro de Estudios Mayas, UNAM

ISSN: 0185-2574 
guientes tendencias: en la primera fase $(600$ a.C.-250 d.C.), que abarca mucho más de la mitad del tiempo en la escala temporal de Uaxactún, se presentaron sólo alrededor de $30 \%$ de todas las innovaciones, mientras que durante el siguiente periodo ( 250 a.C.-378 d.C.), que abarcó sólo menos de $9 \%$ del tiempo en la escala temporal, ocurrió más de $20 \%$ de todas las innovaciones. En los periodos siguientes se observa un número relativamente bajo de innovaciones comparado con la duración de dichas fases (Tzakol 3 y Tepeu 1-2). Pero durante la última etapa del desarrollo cultural en Uaxactún se observa, de nuevo, un alto número de innovaciones. Considerando los datos en los cuadros $5 \mathrm{a}$ y 5 b, se puede deducir que en el subsistema relacionado con la elaboración de cerámica, las fases Tzakol 1, Tzakol 2 y Tepeu 3 marcan los periodos de mayor inestabilidad en comparación con las demás fases.

En Altar de Sacrificios se nota el siguiente patrón (véase cuadro 2). Después de arrancar en Xe se observa una tendencia hacia el estancamiento que se prolonga hasta Salinas. Luego sucede el periodo de crecimiento acelerado de innovaciones, lo que cae en Ayn y Veremos, y desde Chixoy se nota una tendencia hacia un estancamiento. En Pasión sucede otra etapa de crecimiento que se prolonga hasta Jimba. Estas fases separadas se ven en los cuadros 5 a y 5 b. Cabe observar que los dos últimos ciclos: Ayn-Chixoy y PasiónJimba son mucho más dinámicos que los ciclos anteriores que ocupan sin embargo casi $73 \%$ del tiempo en la escala temporal del sitio.

En Seibal ocurren cosas semejantes (véase cuadro 3). Después del arranque en Real se nota una tendencia al estancamiento hasta Cantutsé y posiblemente un inicio de un arranque nuevo en Junco, sin embargo cortado abruptamente alrededor de 500 d.C. Un nuevo arranque del crecimiento sucede en Tepejilote, seguido por una fase de crecimiento acelerado. Ahora bien, los datos de los cuadros $5 \mathrm{a}$ y $5 \mathrm{~b}$ parecen resaltar que existen en realidad dos ciclos en la aparición de innovaciones: desde Real hasta Junco y Tepejilote-Bayal.

De Piedras Negras provienen datos muy limitados. Parecen indicar una tendencia hacia el crecimiento acelerado desde Naba hasta Chacalhaaz (véanse cuadros 4 , 5a y 5b).

Los ciclos de crecimiento acelerado de innovaciones y de su saturación no coinciden exactamente en cada uno de los sitios investigados. Mientras que el primer ciclo termina en Uaxactún ca. 250 d.C.; en Altar de Sacrificios es hasta 450 d.C. cuando éste se acaba; en Seibal se prolonga probablemente hasta 500 d.C., y posiblemente en Piedras Negras su terminación coincide con esta última fecha. El segundo ciclo empieza más temprano en Uaxactún, donde se acaba alrededor de 550; en Altar de Sacrificios, cerca de 630; en Seibal, probablemente Junco podría indicar este ciclo, pero aparentemente este ciclo se terminó de manera muy abrupta, y en Piedras Negras no está muy clara su presencia. En Uaxactún el tercer ciclo arranca en 550 y se termina en 889; en Altar de Sacrificios se inicia en 630 y termina 950; en Seibal este ciclo se ubica entre 650 y 930 , y en Piedras Negras la situación no es muy clara.

Se nota inmediatamente que el mayor número de innovaciones en cerámica suele presentarse en periodos muy cortos, mientras que durante los periodos largos el número de innovaciones tiende a disminuir. Tomando en cuenta lo que se ha dicho arriba, los periodos cortos con un mayor número de innovaciones son los periodos de menor estabilidad, y con un número menor de innovaciones son los periodos de mayor estabilidad. Aquí me limito solamente a la estabilidad mayor y/o menor en el subsistema relacionado con la cerámica. Naturalmente hay que investigar las innovaciones en otros subsistemas culturales. 


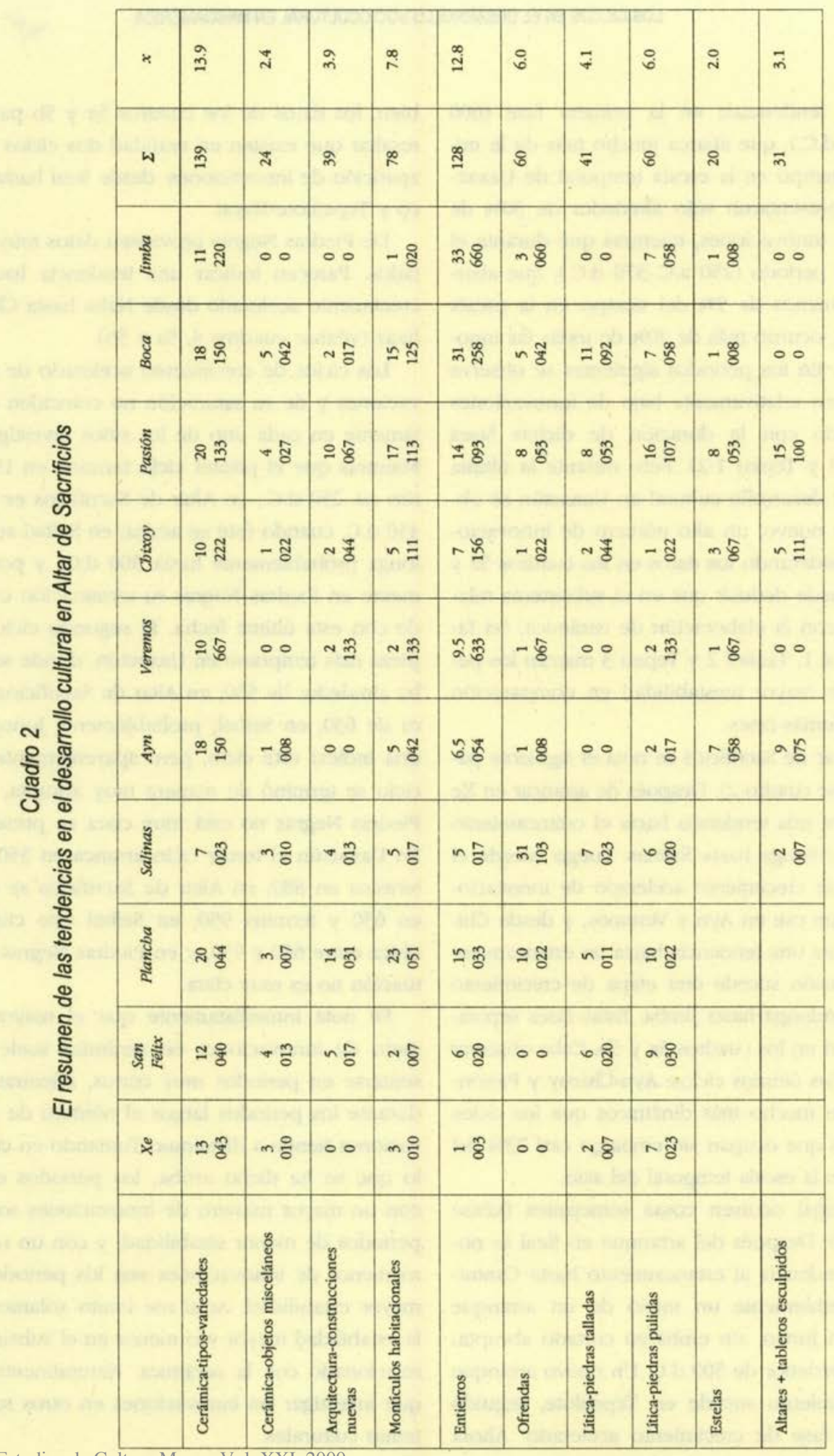

Estudios de Cultura Maya. Vol. XXI, 2000

Instituto de Investigaciones Filológicas/

Centro de Estudios Mayas, UNAM

ISSN: 0185-2574 


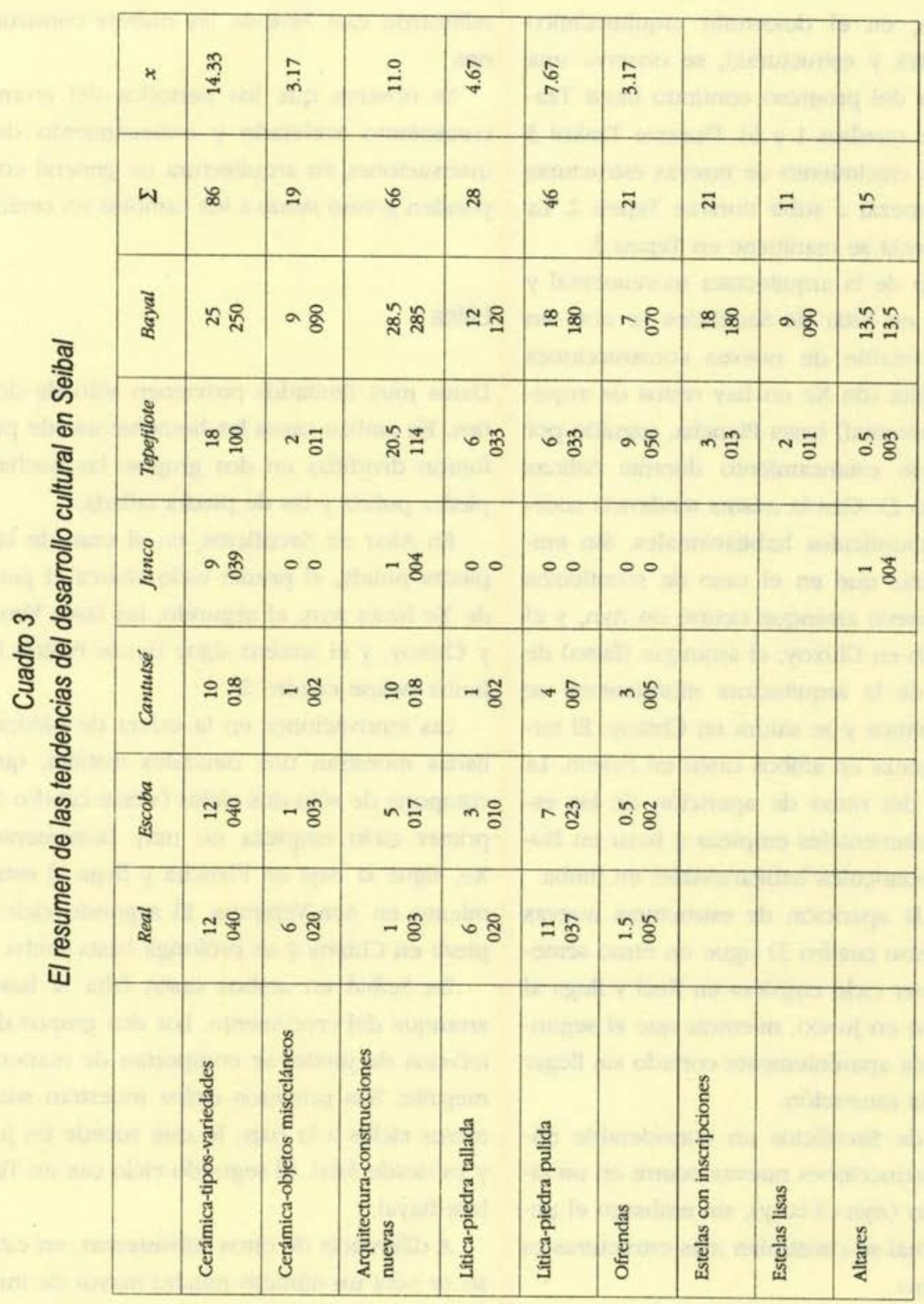

Estudios de Cultura Maya. Vol. XXI, 2000

Instituto de Investigaciones Filológicas/

Centro de Estudios Mayas, UNAM

ISSN: 0185-2574 


\section{Arquitectura}

En Uaxactún, en el desarrollo arquitectónico (construcciones y estructuras), se observa una tendencia fija del progreso continuo hasta Tzakol 2 (véanse cuadros 1 y 6). Durante Tzakol 3 y Tepeu 1 el crecimiento de nuevas estructuras baja para empezar a subir durante Tepeu 2. La misma tendencia se manitiene en Tepeu 3.

En el caso de la arquitectura monumental y habitacional, en Altar de Sacrificios se nota un crecimiento estable de nuevas construcciones desde San Félix (de Xe no hay restos de arquitectura monumental) hasta Plancha, seguido por el periodo de estancamiento durante Salinas (véase cuadro 2). Casi la misma tendencia sucede con los montículos habitacionales. Sin embargo, mientras que en el caso de montículos nuevos un nuevo arranque ocurre en Ayn, y el estancamiento en Chixoy, el arranque (falso) de crecimiento de la arquitectura monumental se inicia en Veremos y se satura en Chixoy. El tercer ciclo empieza en ambos casos en Pasión. La disminución del ritmo de aparición de las estructuras monumentales empieza a bajar en Boca, y el de montículos habitacionales en Jimba.

También la aparición de estructuras nuevas en Seibal (véase cuadro 3) sigue un ritmo semejante. El primer ciclo empieza en Real y llega al estancamiento en Junco, mientras que el segundo ciclo queda aparentemente cortado sin llegar a la fase de la saturación.

En Altar de Sacrificios un considerable número de construcciones nuevas ocurre en un ciclo muy corto (Ayn-Chixoy), sin embargo el periodo en el cual se construían más estructuras es el ciclo tercero.

Una inversión interesante de las proporciones en porcentajes del tiempo en la escala local y de innovaciones sucede en Seibal (véase cuadro 6). Mientras que durante $76 \%$ del tiempo de la duración del desarrollo en Seibal se construEstudios de Cultura Maya. Vol. XXI, 2000

Instituto de Investigaciones Filológicas/

Centro de Estudios Mayas, UNAM

ISSN: 0185-2574 yeron solo $26 \%$ de construcciones nuevas, en el periodo que abarca tan sólo $15 \%$ del tiempo se edificaron casi $74 \%$ de las nuevas construcciones.

Se observa que los periodos del arranque, crecimiento acelerado y estancamiento de las innovaciones en arquitectura en general corresponden grosso modo a los cambios en cerámica.

\section{Lítica}

Datos muy limitados provienen sólo de dos sitios. En ambos casos las herramientas de piedra fueron divididas en dos grupos: las hechas de piedra pulida y las de piedra tallada.

En Altar de Sacrificios, en el caso de las de piedra pulida, el primer ciclo abarca el periodo de Xe hasta Ayn; el segundo, las fases Veremos y Chixoy, y el tercero sigue desde Pasión hasta Jimba (véase cuadro 2).

Las innovaciones en la esfera de piedras talladas muestran una dinámica distinta, que se compone de sólo dos ciclos (véase cuadro 2). El primer ciclo empieza no muy firmemente en $\mathrm{Xe}$, sigue la baja en Plancha y llega al estancamiento en Ayn-Veremos. El segundo ciclo empieza en Chixoy y se prolonga hasta Jimba.

En Seibal en ambos casos falta la fase del arranque del crecimiento. Los dos grupos de artefactos de piedra se comportan de manera semejante. Sus primeros ciclos muestran sus primeros ciclos a la baja, lo que sucede en Junco, y ya desde Real. El segundo ciclo cae en Tepejilote-Bayal.

A diferencia de otros subsistemas, en este caso se nota un número mucho mayor de innovaciones en los primeros ciclos del desarrollo cultural (véase cuadro 7). Sin embargo, las tendencias generales en la aparición de innovaciones, parecen muy semejantes a las de la cerámica y la arquitectura. 


\section{Cuadro 4}

Algunas tendencias en el desarrollo cultural de Piedras Negras

\begin{tabular}{|c|c|c|c|c|c|c|c|c|}
\hline & Preclásico & Naba & Balcbé & Yaxcbé & Cbacalbaaz & Kumcbé & $\Sigma$ & $x$ \\
\hline Cerámica-tipos-variedades & 7 & $\begin{array}{c}18 \\
072\end{array}$ & $\begin{array}{c}22 \\
282 \\
088\end{array}$ & $\begin{array}{r}34 \\
330\end{array}$ & $\begin{array}{r}52 \\
525\end{array}$ & 15 & 148 & 24.67 \\
\hline Estelas & & & $\begin{array}{c}2 \\
026\end{array}$ & $\begin{array}{r}18 \\
175\end{array}$ & $\begin{array}{r}12 \\
121\end{array}$ & & & \\
\hline
\end{tabular}

\section{Cuadro 5a}

Las tendencias en el desarrollo de cerámica. Los tipos-variedades nuevos

\begin{tabular}{|c|c|c|}
\hline Fase arqueológica & $\begin{array}{l}\text { Porcentaje del tiempo } \\
\text { en la escala del sitio }\end{array}$ & $\begin{array}{c}\text { Porcentaje de nuevos } \\
\text { tipos-variedades }\end{array}$ \\
\hline \multicolumn{3}{|c|}{ UAXACTÚN } \\
\hline Mamón (600-300 a.C.) & $20.15 \%$ & $11.41 \%$ \\
\hline Chicanel (300 a.C. -250 d.C.) & $36.94 \%$ & $13.42 \%$ \\
\hline Tzakol 1 (250-300 d.C.) & $3.36 \%$ & $5.37 \%$ \\
\hline Tzakol 2 (300-378 d.C.) & $4.84 \%$ & $8.72 \%$ \\
\hline Tzakol 3 (378-550 d.C.) & $11.55 \%$ & $6.04 \%$ \\
\hline Tepeu 1 (550-692 d.C.) & $9.54 \%$ & $11.41 \%$ \\
\hline Tepeu 2 (692-830 d.C.) & $9.27 \%$ & $22.82 \%$ \\
\hline Tepeu 3 ( $830-889$ d.C.) & $3.96 \%$ & $20.81 \%$ \\
\hline \multicolumn{3}{|c|}{ ALTAR DE SACRIFICIOS } \\
\hline $\mathrm{Xe}(900-600$ a.C. $)$ & $16.22 \%$ & $9.35 \%$ \\
\hline San Félix $(600-300$ a.C. $)$ & $16.22 \%$ & $8.63 \%$ \\
\hline Plancha (300 a.C. -150 d.C.) & $24.32 \%$ & $14.39 \%$ \\
\hline Salinas $(150-450$ d.C. $)$ & $16.22 \%$ & $5.04 \%$ \\
\hline Ayn $(450-570$ d.C.) & $6.49 \%$ & $12.95 \%$ \\
\hline Veremos (570-585 d.C.) & $0.81 \%$ & $7.19 \%$ \\
\hline Chixoy (585-630 d.C.) & $2.43 \%$ & $7.19 \%$ \\
\hline Pasión (630-780 d.C.) & $8.11 \%$ & $14.39 \%$ \\
\hline Boca $(780-900$ d.C. $)$ & $6.49 \%$ & $12.95 \%$ \\
\hline Jimba (900-950 d.C.) & 2. $70 \%$ & $7.91 \%$ \\
\hline
\end{tabular}

Estudios de Cultura Maya. Vol. XXI, 2000

Instituto de Investigaciones Filológicas/

Centro de Estudios Mayas, UNAM

ISSN: 0185-2574 
Cuadro 5 a

(Continúa)

\begin{tabular}{|l|c|c|}
\hline Fase arqueológica & $\begin{array}{c}\text { Porcentaje del tiempo } \\
\text { en la escala del sitio }\end{array}$ & $\begin{array}{c}\text { Porcentaje de nuevos } \\
\text { tipos-variedades }\end{array}$ \\
\hline \multicolumn{3}{|c|}{ SEIBAL } \\
\hline Real (900-600 a.C.) & $16.39 \%$ & $13.95 \%$ \\
\hline Escoba (600-300 a.C.) & $16.39 \%$ & $13.95 \%$ \\
\hline Cantutsé (300 a.C.-270 d.C.) & $31.15 \%$ & $11.63 \%$ \\
\hline Junco (270-500 d.C.) & $12.57 \%$ & $10.47 \%$ \\
\hline Tepejilote (650-830 d.C.) & $9.84 \%$ & $20.93 \%$ \\
\hline Bayal (830-930 d.C.) & $5.46 \%$ & $29.07 \%$ \\
\hline \multicolumn{3}{|c|}{ PIEDRAS NEGRAS } \\
\hline Preclásico & $14.72 \%$ & $4.73 \%$ \\
\hline Naba (300-500 d.C.) & $19.43 \%$ & $14.29 \%$ \\
\hline Balché (550-628 d.C.) & $18.68 \%$ & $17.46 \%$ \\
\hline Yaxché (628-731 d.C.) & & $26.98 \%$ \\
\hline Chacalhaaz (731-830 d.C.) & & $41.27 \%$ \\
\hline Kumché (830-?) & & $10.14 \%$ \\
\hline
\end{tabular}

\section{Cuadro $5 b$}

Las tendencias en el desarrollo de la cerámica

\begin{tabular}{|c|c|c|}
\hline Fase arqueológica & $\begin{array}{l}\text { Porcentaje del tiempo } \\
\text { en la escala del sitio }\end{array}$ & $\begin{array}{c}\text { Porcentaje de nuevos } \\
\text { elementos }\end{array}$ \\
\hline \multicolumn{3}{|c|}{ UAXACTUN } \\
\hline Mamón-Chicanel (600 a.C.-250 d.C.) & $57.09 \%$ & $31.52 \%$ \\
\hline Tzakol 1-Tzakol 2 (250-378 d.C.) & $8.60 \%$ & $22.08 \%$ \\
\hline Tzakol 3 (378-550 d.C.) & $11.55 \%$ & $8.97 \%$ \\
\hline Tepeu 1-Tepeu 3 (550-889 d.C.) & $18.80 \%$ & $18.77 \%$ \\
\hline Tepeu 3 ( $830-889$ d.C.) & $3.96 \%$ & $18.65 \%$ \\
\hline \multicolumn{3}{|c|}{ ALTAR DE SACRIFICIOS } \\
\hline Xe-Plancha (900 a.C. -150 d.C.) & $59.76 \%$ & $33.74 \%$ \\
\hline Salinas ( $150-450$ d.C.) & $16.22 \%$ & $4.91 \%$ \\
\hline Ayn-Chixoy (450-630 d.C.) & $9.73 \%$ & $24.54 \%$ \\
\hline Pasión-Jimba (630-950 d.C.) & $17.30 \%$ & $35.58 \%$ \\
\hline \multicolumn{3}{|c|}{ SEIBAL } \\
\hline Real-Junco (900 a.C.-500 d.C.) & $76.50 \%$ & $48.57 \%$ \\
\hline Tepejilote-Bayal (650-930 d.C.) & $15.30 \%$ & $51.43 \%$ \\
\hline
\end{tabular}

Instituto de Investigaciones Filológicas/

Centro de Estudios Mayas, UNAM

ISSN: 0185-2574 


\section{Cuadro 6}

Las tendencias en el desarrollo de la arquitectura

\begin{tabular}{|l|c|c|}
\hline Fase arqueológica & $\begin{array}{c}\text { Porcentaje del tiempo } \\
\text { en la escala del sitio }\end{array}$ & $\begin{array}{c}\text { Porcentaje de nuevos } \\
\text { elementos }\end{array}$ \\
\hline \multicolumn{2}{|c|}{ UAXACTÚN } \\
\hline Mamón-Chicanel (600 a.C.-250 d.C.) & $57.09 \%$ & $20.78 \%$ \\
\hline Tzakol 1-Tzakol 2 (250-378 d.C.) & $8.60 \%$ & $50 \%$ \\
\hline Tzakol 3 (378-550 d.C.) & $11.55 \%$ & $12.99 \%$ \\
\hline Tepeu 1-Tepeu 3 (550-889 d.C.) & $22.76 \%$ & $16.23 \%$ \\
\hline \multicolumn{2}{|c|}{ ALTAR DE SACRIFICIOS } \\
\hline Xe-Plancha (900 a.C.-150 d.C.) & $59.76 \%$ & $40.17 \%$ \\
\hline Salinas (150-450 d.C.) & $16.22 \%$ & $7.69 \%$ \\
\hline Ayn-Chixoy (450-630 d.C.) & $9.73 \%$ & $13.68 \%$ \\
\hline Pasión-Jimba (630-950 d.C.) & $17.30 \%$ & $44.44 \%$ \\
\hline Real-Junco (900 a.C.-500 d.C.) & $76.50 \%$ & $25.76 \%$ \\
\hline Tepejilote-Bayal (650-930 d.C.) & $15.30 \%$ & $74.24 \%$ \\
\hline
\end{tabular}

Cuadro 7

Las tendencias en el desarrollo de la lítica

\begin{tabular}{|l|c|c|}
\hline Fase arqueológica & $\begin{array}{c}\text { Porcentaje del tiempo } \\
\text { en la escala del sitio }\end{array}$ & $\begin{array}{c}\text { Porcentaje de nuevos } \\
\text { elementos }\end{array}$ \\
\hline \multicolumn{2}{|c|}{ ALTAR DE SACRIFICIOS } \\
\hline Xe-Plancha (900 a.C.-150 d.C.) & $56.76 \%$ & $38.61 \%$ \\
\hline Salinas (150-450 d.C.) & $16.22 \%$ & $12.87 \%$ \\
\hline Ayn-Chixoy (450-630 d.C.) & $9.73 \%$ & $6.93 \%$ \\
\hline Pasión-Jimba (630-950 d.C.) & $17.30 \%$ & $41.58 \%$ \\
\hline & SEIBAL & \\
\hline Real-Junco (900 a.C.-500 d.C.) & $76.50 \%$ & $41.89 \%$ \\
\hline Tepejilote-Bayal (650-930 d.C.) & $15.30 \%$ & $56.76 \%$ \\
\hline
\end{tabular}

Estudios de Cultura Maya. Vol. XXI, 2000

Instituto de Investigaciones Filológicas/

Centro de Estudios Mayas, UNAM

ISSN: 0185-2574

http://www iifilologicas.unam.mx/estculmaya/ 


\section{Conclusiones}

En el presente estudio sobre el ritmo de la aparición de innovaciones faltan naturalmente muchos más datos para trazar las trayectorias del desarrollo cultural en sitios respectivos. A estos datos hay que agregar los cálculos de grado de organización de las variables culturales mencionadas. La falta de espacio me impidió aquí profundizar en este enfoque. Pero lo hice en otro trabajo (Iwaniszewski, 1988). Aquí intenté solamente presentar la necesidad de estudiar las innovaciones.

Aunque las variables escogidas para el presente trabajo son todavía muy preliminares y poco exactas, demuestran, sin embargo, un comportamiento semejante. El hecho de que las tendencias en el crecimiento de innovaciones en un subsistema corresponden a las del otro refuerza la hipótesis señalada al principio: muchos cambios culturales ocurren en periodos cortos, mientras que pocos cambios ocurren en periodos largos; de acuerdo con este enfoque, estos periodos cortos son las épocas de mayor inestabilidad del sistema y los periodos largos, caracterizados por el estancamiento, corresponden a las épocas de mayor estabilidad del sistema.

Se nota que estos periodos se presentaron en todos los sitios analizados, pero no coincidieron con respecto a sí mismos. Cada sitio sigue su desarrollo propio, a pesar de que vistos en una perspectiva global pasan por fases semejantes en los mismos periodos de tiempo.

\section{Bibliografia}

Ammerman, A. J., y L. L. Cavalu-Sforza

1973 "A population model for the diffusion of early farming in Europe", en Colin Renfrew (ed.), The Explanation of Culture Change: Models in Prebistory, University of Pittsburgh Press, Pittsburgh, pp. 343-358.

Cavalu-Sforza, L. L., y M. W. Feldman

1981 Cultural transmission and Evolution: $A$ Quantitative Approach, Princeton University Press, Princeton.

Crane, Duna

1972 Invisible Colleges, The University of Chicago Press, Chicago.

Hamblin, Robert L., R. BRooke JACobsen y JerRy L. L. MILLER

1973 A Mathematical Theory of Social Change, Wiley, Nueva York.

IwanISZEwWSKI, StanISLAw

1988 El papel de la astronomía en el desarrollo cultural en Mesoamérica, tesis de doctorado, Universidad Nacional Autónoma de México.

Lenski, Gerhard, y Jean Lenski

1974 Human Societies, McGraw-Hill, Nueva York. LUENSBERger, David G.

1979 Introduction to Dynamic Systems. Theory, Models, and Applications, Wiley, Nueva York.

Price, Derek de Solla

1978 Science since Babylon, Yale University Press, New Haven.

ReNFrew, Colin

1984a "Systems Thinking: The Exploration of Continuous Change", en Colin Renfrew (ed.), $A p$ proaches to Social Arcbaeology, Harvard University Press, Cambridge, Mass., pp. 248257.

1984b "Culture Systems and the Multiplier Effect", en Colin Renfrew, Approacbes to Social Archaeology, Harvard University Press, Cambridge, Mass., pp. 258-282.

Renfrew, Coun, y Kenneth L. CoOKe

1984 "An Experiment on the Simulation of Culture Changes", en C. Renfrew, Approaches to Social Archaeology, Harvard University Press, Cambridge, Mass., pp. 309-330.

StarR, Chauncey, y Richard Rudman

1973 "Parameters of Technological Growth", Science, 182, 4110: 358-364. 